\title{
Los instagramers más influyentes de Ecuador
}

\section{The most influencer instagramers from Ecuador}

\author{
Erika-Lucía González-Carrión \\ Universidad Nacional de Loja, Ecuador \\ erikalucia@hotmail.es \\ https://orcid.org/0000-0003-3808-5460 \\ Ignacio Aguaded \\ Universidad de Huelva, España \\ ignacio@aguaded.es \\ https://orcid.org/0000-0002-0229-1118
}

\begin{abstract}
Resumen
Instagramers en un contexto mediático es un tema en pleno auge, del cual poco se ha escrito entre la comunidad científica pero paulatinamente está tomando mayor importancia dado que a corto plazo Instagram apunta a ser una de las redes sociales favoritas y más utilizadas. A través de esta investigación se pretende identificar cuáles son los instagramers más influyentes en Ecuador, así como analizar los perfiles de los mismos en función de un análisis de contenidos (producción y tecnología), análisis de lenguaje (lenguaje) y el feedback / interacción (difusión) generada desde sus perfiles. La metodología empleada ha sido cualitativa y cuantitativa, a través de observación no participante, la herramienta Alianzo Raking y una entrevista semiestructurada a un experto en el tema de estudio. Los resultados muestran que el nivel de respuesta a los comentarios de los seguidores, las publicaciones que generan auto-identificación y sentimiento de cercanía con las audiencias, las fotografías de mascotas (perros) publicados por parte de los Instagramers exitosos son aceptadas por la comunidad seguidores y generan mayor cantidad de "me gusta". El análisis de los instagramers exitosos dentro de Ecuador sirve para realizar una evaluación y detectar qué interesa a la gente en un entorno social determinado, además de las causas que llevan a un instagramer tener mayor éxito sobre otro.
\end{abstract}

\section{Palabras clave}

Instagram, instagramers, competencia mediática, seguidores, audiencia, éxito.

Forma sugerida de citar: González-Carrión, E., y Aguaded, I. (2019). Los instagramers más influyentes de Ecuador. Universitas, 31, pp. 159-174. 


\begin{abstract}
Instagramers in the context of media literacy is a booming theme, of which little has been written among the scientific community but gradually is taking greater importance because in a short term Instagram aims to be one of the most used and favorite social networks. Through this research, it is intended to determine which are the most influencer instagramers in Ecuador, as well as to study their profiles according to content analysis (production and technology), analysis of language (language) and feedback / interaction (diffusion) generated since their profiles. The methodology used has been qualitative and quantitative through non-participant observation, Alianzo Ranking tool and a semi-structured interview applied to an expert in the study. The results show that the level of response to the comments of the followers, the publications that generate self-identification and feeling of closeness to the audience, the photographs of pets (dogs) posted by the successful instagramers are accepted by the community of followers and generate more "likes". The analysis of successful instagramers in Ecuador serves to make an evaluation and detect what interests to people in a particular social environment and thus the causes that lead to one instagramer to succeed over another.
\end{abstract}

Keywords

Instagram, instagramers, media literacy, followers, audience, success.

\title{
Introducción y estado de la cuestión
}

La comunicación, por su naturaleza ha estado presente junto a los seres humanos desde el inicio de los tiempos y ha ido evolucionando paulatinamente a la par de los avances tecnológicos de un mundo tan globalizado en el que vivimos. Si anteriormente nos comunicábamos a través de señales de humo, contacto físico o sonidos guturales ahora la comunicación ha dado un giro total a tal punto que las distancias están tan solo un clic. Ahora es posible visualizar a personas del otro lado del mundo en tiempo real al igual que compartir momentos a través de imágenes, como es el caso de las redes sociales.

Así, Lacoste (1998) sostiene:

(...) Internet es una fuente de recursos de información compartidos a escala mundial. Es una vía de comunicación para establecer cooperación y colaboración entre comunidades y grupos de interés por temas específicos, distribuidos por todo el mundo. (p. 182) 
De hecho, cuando ingresamos a la World Wide Web nos conectamos con personas de todo el globo terráqueo, independientemente de su ubicación geográfica, y luego a través de las conexiones de red realizadas podemos lograr establecer contactos que bien puede ser de utilidad para formar relaciones sociales, para re-encontrarse con amistades del ayer, para elaborar proyectos e inclusive para trabajar y estudiar.

Las redes sociales en los últimos años han tenido un auge de gran magnitud y en la actualidad se han convertido en parte de nuestro diario convivir social, y son un patrón fundamental de vida (Castells, 2009), pues cada día va en aumento el número de usuarios a nivel global que crean, interactúan y comparten en cada segundo sus vivencias a través de su red de preferencia. Algunos los realizan con fines de transmitir visualmente perfiles únicos a través del lenguaje fotográfico, otros por el hecho de anhelar ser más populares o hacer comparaciones sociales (Utz, Tanis, \& Vermeulen, 2012) y hay quienes a través de plataformas basadas en imágenes como Snapchat e Instagram logran disminuir su soledad e incrementan sus niveles de felicidad y satisfacción con la vida (Pittman \& Reich, 2016).

En este entorno surge Instagram, red social y aplicación para subir fotos y videos Frommer (citado por Sheldon \& Bryant, 2016), pero en el sitio adicionalmente el individuo puede editar a las fotografías con diferentes filtros, marcos, colores, e infinidad de recursos a disposición del usuario según sus gustos y más variadas preferencias. Luego, una vez editadas las fotografías, las mismas son subidas a través de la misma red y son compartidas con los amigos virtuales quienes proveen cierta retroalimentación a las fotografías publicadas ya sea con comentarios o haciendo clic en el botón de "Me gusta".

A quienes se le atribuye el hecho de la creación de Instagram es a Kevin Systrom y Mike Krieger en el año 2010, y desde entonces el éxito paulatino que esta red ha tenido es cada vez a escala mayor, y todo apunta a que su crecimiento mejorará día a día. Así por ejemplo se puede citar a los datos de la página Shareaholic, en donde se hace referencia al hecho de que durante el lapso de julio a diciembre de 2017 la cantidad de tráfico que Instagram aportaba a las páginas web de todo el mundo ganó una importancia notable de $0,1 \%$ a un $0,7 \%$.

Esta red social/aplicación es un claro ejemplo del avance de las redes sociales y las tecnologías 2.0. porque se han creado comunidades virtuales que sienten la necesidad de compartir y comunicar. Así, éstas nuevas redes han 
traído consigo la necesidad de utilizar nuevos lenguajes que implican mucho más del usuario, quien ya no es más un ente pasivo sino activo, capaz de producir contenidos, compartirlos y darlos a conocer a su entorno virtual como se podrá evidenciar más adelante.

Instagram es una red social que como se ha mencionado anteriormente permite muchas formas de participación por parte de los usuarios, quienes pueden reflejar a través de esta red sus formas de pensar, actuar, aptitudes, habilidades, entre otros.

En este apartado se dedicará algunas líneas a explicar los resultados de varias investigaciones en torno a esta temática, lo cual nos permitirá tener un panorama más claro de lo que acontece en torno a esta red social. Se explicará por ejemplo la manera a través de la cual el insertar fotografías en Instagram puede llevar a promover conductas narcisistas y cómo puede provocar que la gente se sienta influenciada por cómo otras personas los perciben y juzgan (Jin \& Muqaddam, 2018) y de manera muy similar se explica que usuarios de esta red la utilizan para vigilar, documentar y conocer sobre los otros y demostrar su creatividad, viajes y actividades de ocio (Sheldon \& Bryant, 2016).

Por otro lado, se observará cómo tras una investigación realizada entre jóvenes adultos se logró determinar que con el uso de Instagram este grupo que ha sido catalogado como el más solitario de la generación logra reducir sus niveles de soledad y sus niveles de felicidad y satisfacción con la vida se incrementan paulatinamente (Pittman \& Reich, 2016). Si en cambio, analizamos las conductas de adolescentes durante sus interacciones en Instagram, logramos determinar que algunos ellos buscan la aprobación de sus pares y comparten contenidos con el fin de parecer atractivos e interesantes y previo a la publicación de fotografías en Instagram deben seguir según su parecer "normas" para lo cual en ciertos casos se ven en la necesidad de pedir ayuda (Yau \& Reich, 2018).

De manera similar se revisará de manera breve la influencia que tienen los "likes" me gusta dentro de Instagram a nivel cerebral, en un estudio a través del cual se observó que ciertas zonas cerebrales se activan en función de la retroalimentación recibida por el sujeto con los "me gusta" dados por sus pares virtuales (Sherman, Greenfield, Hernandez, \& Dapretto, 2017). De manera adicional se observará cómo la forma de presentación de las imágenes en Instagram puede implicar credibilidad o es posible que incite a la compra a los usuarios (Colliander \& Marder, 2018) o más aún cómo incre- 
mentan en las mujeres las intenciones de compra a través de la visualización de celebridades en Instagram (Djafarova \& Rushworth, 2017).

a) Instagram y narcicismo: Jin y Muqaddam (2018) en su estudio demuestran a través de dos experimentos cómo el hecho de insertar fotografías en Instagram, específicamente selfies (fotografías de sí mismos) implica que el usuario desea auto-promocionarse así como también se indica que de acuerdo a la fotografía pueden ponerse de manifiesto emociones narcisistas en el sujeto, en quien también se muestra la necesidad de popularidad. Por otro lado, se toma muy en cuenta la personalidad de los sujetos, la que se constituye en un factor clave dentro del narcisismo el mismo que se define en función del número de visualizaciones de las fotografías posteadas y el número de seguidores.

b) Instagram para vigilar y documentar: Sheldon y Bryant (2016) explican en su estudio que Instagram es la red social con crecimiento más rápido a nivel global y logran determinar que la principal razón de los usuarios para utilizar Instagram, que difiere de redes sociales similares, es la "vigilancia/conocimiento" sobre los otros, Se encontró además que las mujeres son más activas en Instagram que los hombres, además que ellas se comunican más con sus amistades que el sexo opuesto. Adicionalmente los autores explican que cuando las mujeres comentan las fotografías de sus amigos, ellas están enviando un mensaje de que a ellas "les importan". De manera similar al estudio citado previamente, se determinó que Instagram resulta muy atractivo para desarrollar actitudes narcisistas y que diferentes circunstancias sociales y psicológicas (satisfacción social, actividad social, narcisismo) pueden reforzar diferentes tendencias conductuales que a su vez desencadenan como resultado diferentes conductas (número de horas en el sitio, edición de fotos, uso de hashtags).

c) Instagram para reducir la soledad y aumentar los niveles de felicidad: Pittman y Reich (2016) a través de su investigación demuestran cómo las redes sociales basadas en la imagen como Snapchat e Instagram contribuyen a disminuir la soledad en las personas, en una sociedad donde la población de jóvenes adultos sufre la soledad más fuerte de todos los tiempos. Mientras una plataforma social sea más basada en la imagen que en el texto, los usuarios se sentirán más felices, más satisfechos con la vida y el o ella se percibirán menos solitarios. Las imágenes facilitan la presencia social, el sentido de que se establece una comunicación con una persona en lugar de con un objeto. La foto de un amigo comiendo o haciendo alguna cara 
divertida- o inclusive si es un video y su voz se puede escuchar- es más probable que ésta sea una señal para el cerebro de que el amigo está ahí, efectos que no se provocan con las redes sociales basadas en el texto.

d) Instagram como espejo de la persona ¿me veo atractivo?: Yau y Reich (2018) pretendían a través de su estudio determinar las normas de presentación que siguen los adolescentes antes de colocar contenido "posts" a través de las redes sociales Facebook e Instagram. Los resultados indican que los adolescentes previo a realizar alguna publicación en las redes en mención buscan la aprobación de sus pares para verse atractivos e interesantes. Inclusive en el caso de las adolescentes es mayormente marcado el hecho de preguntar a sus amigos sus criterios sobre cierto post previa publicación del mismo, por lo que se ha observado que lo que en un inicio resultaba una actividad de entretenimiento en ciertos casos puede ser tediosa. Los adolescentes consideran que es de gran importancia seguir algunas normas de comportamiento en línea previa publicación de posts, lo cual requiere cierta competencia social.

e) Instagram y la influencia de likes en el cerebro: Sherman, Greenfield, Hernandez, y Dapretto (2017) indican en su estudio que una de las principales características de las redes sociales es la posibilidad de colocar "me gusta" en el contenido posteado por los pares virtuales, por ello este grupo de investigadores examinaron las respuestas conductuales y neurales de los individuos (adolescentes y jóvenes adultos) observando sus fotografías y las de otros mientras se sometían a imágenes de resonancia magnética funcional. Los participantes sentían una atracción especial por aquellas fotografías que habían recibido mayor número de "me gusta", y las fotografías más populares provocaron mayor actividad en múltiples regiones cerebrales, incluyendo el núcleo accumbens, al cual se le atribuye una función importante en el pacer, incluyendo la risa y la recompensa, así como el miedo (Schwienbacher, Fendt, Richardson, \& Schnitzler, 2004), por lo que se encuentra implicado en el circuito de premio- recompensa. El nivel de respuesta de éste núcleo cerebral se activaba mayormente de acuerdo a la edad de los participantes.

f) Instagram como motivante de compras: Colliander y Marder (2018) explican en su estudio que al haber utilizado dos probabilidades a) mostrar a los individuos fotografías sin mayor estética, que podían bien haber sido captadas por un usuario promedio y b) colocar fotografías trabajadas con mayor estética, en ambos casos relacionadas a una marca de moda durante 
el lapso de una semana. Como resultado se obtuvo que las imágenes mayormente elaboradas captaban en mayor grado la atención de los usuarios, quienes a su vez recomendaban a otros seguir la cuenta de Instagram en mención e incrementaban a su vez, por la estética de la imagen, la credibilidad en la fuente. De manera similar Djafarova y Rushworth (2017) corroboran con otros autores que en Instagram la mayor parte de sus usuarios son mujeres jóvenes y en su estudio determinan que las celebridades en Instagram son influenciadoras en las conductas de compra de este grupo de mujeres.

Todos estos cambios conllevan nuevos vocablos que poco a poco se van insertando en nuestro diario comunicar, tal es el caso del término infoxicación, el cual "surge de la unión de las palabras información más intoxicación" y hace referencia a la idea de la sobrecarga de información que recibe un usuario, en especial de internet, en todas sus formas, la cual a su vez puede causar la sensación de no poder abarcar ni gestionarla, y por lo tanto llegar a generar una gran angustia (Toffler, 1970).

Romero-Rodríguez y Aguaded (2016) definen a la infoxicación como "un exceso de consumo de información innecesaria para la toma de decisiones- mayoritariamente contenidos de entretenimiento- es decir, sobresaturación de la capacidad cognitiva de un individuo que acarrea distracción de los asuntos de importancia y su propia alienación racional”.

Basándose en el contexto de la competencia mediática, se plantea la interrogante, ¿los instagramers son prosumidores? Un prosumidor es aquella persona que "crea bienes, servicios o experiencias y simultáneamente está implicado en la producción y consumo de los mismos" (Toffler, 1970). Como se puede evidenciar el término prosumers ha sido empleado desde hace ya mucho tiempo y ha tomado aún más fuerza con la proliferación de Internet, el cual pone de actualidad el "prosumo", en donde la Web 2.0 es concebida como una nueva fábrica social (Ritzer, Dean, \& Jurgenson, 2012). Si tomamos como punto de análisis del prosumismo a las redes sociales la premisa básica es crear y compartir, entonces si la persona no comparte, la red social no fluye, no tiene sentido. Los usuarios realizan el proceso de consumo y producción a través de las nuevas tecnologías, blogs y redes sociales donde existen dinámicas de lectura, retroalimentación y producción de contenidos.

Berrocal, Campos-Domínguez, y Redondo (2014) explican que: 
Las redes sociales son claramente un medio de 'prosumo' donde los prosumidores se convierten en altavoces que producen conversaciones con y para un público consumidor (...) significa que cualquier actividad en el mundo off-line puede tener transcendencia en el mundo on- line, conformando un 'espacio de autonomía'. (pp. 65-72)

La llegada del smartphone cambió por completo el panorama digital, se puede mencionar a Wang, Xiang, y Fesenmaier (2016) quienes en su investigación realizada sobre el tema logran determinar que los smartphones son utilizados para fines de comunicación (llamadas, mensajes de texto, correos electrónicos), entretenimiento (música, libros, fotografías y juegos), redes sociales en línea (Facebook, Twitter e Instagram), búsqueda de información y adquisiciones (noticias, información sobre el tiempo, restaurantes, compras, ideas de viaje y búsquedas en general), y con herramientas de organización personal (banco, alarma, calendario y navegación).

En el estudio mencionado se determinó además que los propietarios de smartphones los utilizaban a los mismos durante todo el día, es decir formaban parte de su diario vivir al punto que muchos de los entrevistados mencionaron que por la mañana despertaban con las alarmas de los teléfonos y abrían sus ojos de vez en cuando por la noche para observar algunas notificaciones de las redes sociales y correos electrónicos, luego durante el día lo utilizaban para buscar lugares donde consumir alimentos, realizar compras, entre otros.

Con base en los criterios y bases de estudio planteados, este trabajo de investigación pretende determinar cuáles son los cinco instagramers de mayor impacto en el Ecuador, así como estudiar sus perfiles en función de la actividad que aquí se realiza.

\section{Material y métodos}

La metodología utilizada para el presente trabajo de investigación será cualitativa y cuantitativa, a través de observación no participante y empleando una entrevista semiestructurada al experto en competencias mediáticas, Dr. Joan Ferrés, enfocada en las competencias mediáticas que poseen los Instagramers de éxito y que son objeto de estudio de la presente investigación.

Para el análisis cuantitativo de los perfiles de los instagramers y la selección de las muestras de la presente investigación se tomó como referencia a los cinco instagramers exitosos de Ecuador ¿Cómo determinar su éxito? Para lograrlo 
se optó por utilizar el muy conocido Alianzo Ranking, el cual utiliza una puntuación entre el 0 y el 100 lo cual es el resultado de combinar distintos parámetros de medición para cada uno de los perfiles sociales a los que pertenece cada usuario. Entre los indicadores para que un instagramer figure en el ranking sobre otro usuario constan el número de seguidores y la interacción con los usuarios.

Los criterios seguidos para la elección del ranking de Alianzo han sido los siguientes:

- Posibilita seleccionar los perfiles de los instagramers de éxito haciendo un filtraje por categorías (País, red social, idioma, entre otros).

- Es un ranking actualizado semanalmente que proporciona datos reales de la persona que se está analizando y se muestra estadísticamente el avance en cuanto a seguidores por lapsos desde una semana, un mes, tres meses, seis meses, un año y a nivel global.

- Este ranking ha sido empleado en varias investigaciones científicas, dada su fiabilidad en datos y su gran valor. Así por ejemplo podemos citar a los trabajos realizados por Santoveña (2011), Moya-Sánchez y Herrera-Damas (2016), Tejedor et al. (2009), entre otros.

Con la ayuda de Alianzo Ranking se realizó una selección de los cinco instagramers de mayor éxito en Ecuador tomando en cuenta su influencia dada por el número de seguidores y la interacción generada con los mismos.

Para el análisis de los perfiles de los instagramers que son objeto de estudio se lo realizó en función de las dimensiones establecidas de la competencia mediática que posee un instagramer exitoso a través de sus publicaciones y en función de las opiniones vertidas por el experto se procedió a realizar el análisis de los datos con los siguientes apartados: análisis de contenido, análisis del lenguaje y feedback. Adicionalmente el contenido de la entrevista semiestructurada fue base fundamental tanto para el sustento teórico en la variable de competencias mediáticas, así como para la elaboración de la discusión del presente trabajo investigativo.

\section{Análisis y resultados}

A través de Alianzo Ranking se determina los cinco instagramers que generan mayor repercusión en Ecuador y a través de la observación no participante, es posible establecer su contenido, lenguaje empleado a través de 
las publicaciones y la interacción que entablan con los seguidores que participan de sus contenidos.

\section{Tabla 1}

Cuenta Instagram María Teresa Guerrero

\begin{tabular}{|c|c|c|c|}
\hline \multirow{2}{*}{ María Teresa Guerrero } & Número de seguidores & Seguidos & Publicaciones \\
\cline { 2 - 4 } & 911000 & 2753 & 8247 \\
\hline
\end{tabular}

Fuente: Alianzo Ranking

Análisis de contenido: Según Alianzo Ranking, María Teresa Guerrero es quien lidera la lista de los instagramers con mayor número de seguidores y actividad en la red social en mención. Cuenta con un total de 911000 seguidores, 2753 seguidos y 8247 publicaciones. El tema principal en su perfil de Instagram es su día a día en EE.UU.

Lenguaje: El lenguaje empleado en las publicaciones que realiza esta Instagramer es bastante claro, concreto y conciso, es así que con el mismo logra hacerse entender por los seguidores de su red social. Los hashtags mayormente utilizados por esta Instagramer son \#Nilos, \#Pinchi - los nombres de sus dos perros, \#tsxflacaguerrero- su línea de ropa deportiva.

Feedback: Uno de los aspectos positivos que se evidenció en la recogida de datos es la gran interacción que María Teresa Guerrero mantiene con sus seguidores. Es así que en las publicaciones que se refieren a su línea de ropa deportiva los seguidores obtienen respuestas de parte de la instagramer exitosa, pero no solamente cuando de vender se trata, sino también en otro tipo de publicaciones.

Tabla 2

Cuenta Instagram \#ChocoTrip

\begin{tabular}{|l|c|c|c|}
\hline \#ChokoTrip & Número de seguidores & Seguidos & Publicaciones \\
\cline { 2 - 4 } Viajando por Ecuador & 26500 & 6907 & 4161 \\
\hline
\end{tabular}

Fuente: Alianzo Ranking

Análisis de contenido: Christian Echeverría (chokoec) es quien ocupa el segundo lugar en el ranking de Alianzo, cuenta con un total de 26500 se- 
guidores, 6907 seguidos y 4161 publicaciones. El tema principal en su perfil de Instagram son fotografías de sus viajes y aventuras por los lugares que visita. Así por ejemplo pone de manifiesto su gusto por los paisajes más variados e inclusive extraños.

Lenguaje: En las diferentes publicaciones de chokoec se puede evidenciar que el lenguaje empleado es bastante entendible para los públicos que lo siguen, de manera especial para los amantes de viajes y aventuras. En sus publicaciones utiliza hashtags en los cuales se auto-menciona \#ChokoTrip y también alude hacia el país que se encuentra visitando.

Feedback: Al igual que en el caso anterior se puede decir que la interacción que guarda este individuo hacia sus seguidores puede constituirse en una de las claves de éxito, dado que el usuario que observa que su comentario tiene una retroalimentación se siente satisfecho, motivado y con cierta importancia porque su mensaje ha tenido una respuesta y por ello se destaca entre el grupo.

\section{Tabla 3}

Cuenta Instagram Richard Salazar Barona

\begin{tabular}{|l|c|c|c|}
\hline \multirow{2}{*}{ Richard Salazar Barona } & Número de seguidores & Seguidos & Publicaciones \\
\cline { 2 - 4 } & 55800 & 234 & 362 \\
\hline
\end{tabular}

Fuente: Alianzo Ranking

Análisis de contenido: El caso de Richard Salazar Barona se puede observar que su público objetivo, son adolescentes y es por ello que sus publicaciones se fundamentan en fotografías de sí mismo, en muchos casos tratándose de selfies en los cuales aparece muy atractivo con ropa bastante juvenil. En las publicaciones muestra su día a día y transmite cariño, amor y empatía hacia sus seguidoras, quienes en la mayoría son mujeres.

Lenguaje: Si el punto de análisis es el lenguaje empleado en las publicaciones de Richard Salazar Barona damos cuenta que es el de un adolescente que busca captar la atención de las seguidoras, dado que utiliza frases como: "Hoy me retiraron los brackets y me corte el cabello ¿Quieren que suba una foto de cómo quedé? \#RompeElHielo".

Feedback: YouTube es otro de los medios utilizados por Richard Salazar además de la cuenta de Instagram y a través de sus publicaciones llama a la interacción por parte de los seguidores, o en su mayoría seguidoras. En 
las publicaciones el instagramer escribe mensajes como: "Vamos a perseguir nuestro sueño a la final, vida solo hay una”.

Tabla 4

Cuenta Instagram Dalo Bucaram

\begin{tabular}{|l|c|c|c|}
\hline \multirow{2}{*}{ Dalo Bucaram } & Número de seguidores & Seguidos & Publicaciones \\
\cline { 2 - 4 } & 362000 & 4071 & 4099 \\
\hline
\end{tabular}

Fuente: Alianzo Ranking

Análisis de contenido: Abdalá Jaime Bucaram Pulley es un reconocido político ecuatoriano, oriundo de Guayaquil, quien dentro del ranking de Alianzo ocupa el cuarto lugar ello debido al número de seguidores que llega a un total de 362000,4071 seguidos y 4099 publicaciones. En sus publicaciones se puede evidenciar que en su mayoría son fotografías de su vida privada, es así que capta con su lente a sus hijos, esposa y se realiza selfies.

Lenguaje: A través de las publicaciones de Dalo Bucaram es muy notorio darse cuenta que es una persona muy amante a su familia y creyente en Dios, ello se puede evidenciar por los hashtags que emplea en sus publicaciones tales como \#fuerzayFe, \#lafuerzadelamor.

Feedback: A diferencia de los instagramers mencionados anteriormente se puede mencionar que Dalo Bucaram no mantiene interacción con sus seguidores y frente a los comentarios que escriben sus seguidores no brinda ninguna respuesta y pese a que algunos individuos lo aluden con preguntas o menciones daloxhecho no responde a los seguidores; sin embargo, se observa un gran número de seguidores.

Tabla 5

Cuenta Instagram Guillermo Lasso

\begin{tabular}{|c|c|c|c|}
\hline \multirow{2}{*}{ Dalo Bucaram } & Número de seguidores & Seguidos & Publicaciones \\
\cline { 2 - 4 } & 90800 & 56 & 822 \\
\hline
\end{tabular}

Fuente: Alianzo Ranking

Análisis de contenido: Guillermo Lasso, político ecuatoriano y excandidato a la Presidencia del Ecuador de acuerdo a Alianzo Ranking ocupa el 
quinto lugar de los instagramers ecuatorianos más exitosos. Por otro lado demuestra a través de imágenes las apariciones públicas que tiene en los medios de comunicación o cuando tiene encuentros con ciudadanos. En publicaciones previas cuando Ecuador atravesaba una época electoral se observa publicaciones con temas de campaña, videos, entrevistas, entre otros.

Lenguaje: Si bien es cierto Guillermo Lasso es un político que aspiró en su tiempo ocupar la Presidencia de Ecuador, pero según las publicaciones de Instagram se puede evidenciar que el político en mención aún aspira por un puesto político. Así por ejemplo el lenguaje que emplea en sus publicaciones tiene un tinte orientado a este fin: "Yo le digo a los jóvenes que participen en política, no dejen la silla vacía, y si está llena por indecentes hay que sacarlos con la fuerza de nuestras ideas y nuestra decencia. ¡Que viva un EcuadorLibre!".

Feedback: Al analizar las publicaciones de guillermolasso resulta notorio evidenciar que en los comentarios que los seguidores hacen frente a las publicaciones del instagramer existe muy poca interacción en comparación con otros instagramers analizados; sin embargo, pese a tal hecho el número de seguidores de Lasso es bastante considerable.

Joan Ferrés, destacado doctor y experto en temas de competencias mediáticas afirma:

Yo hablaría de equilibrios. Siempre se ha dicho (y estoy de acuerdo) que la calidad es más importante que la cantidad, pero no puedes prestar un servicio público si no eres capaz de llegar al mayor número posible de personas. Se repite con las nuevas tecnologías y con las nuevas prácticas comunicativas lo que ocurría con las clásicas. Una televisión de gran calidad que interesaba a muy pocos espectadores no prestaba un servicio público, porque hacía como los bancos, sólo daba cultura a los que ya la tenían. El mismo criterio sirve para Instagram y las redes sociales (...).

Se puede coincidir con Joan Ferrés en el hecho de que se debe buscar un punto de equilibrio porque para contar con un amplio número de seguidores es necesario saber qué es lo que a aquel grupo de seguidores le interesaría conocer u observar en su alimentador de noticias de su cuenta de Instagram. He ahí donde radica la importancia de que un instagramer posea dominio en las competencias mediáticas. A través de esta investigación se analizó a cada instagramer exitoso bajo los siguientes criterios, cada uno correspondiente a una dimensión de competencia mediática: análisis de contenido (producción 
y tecnología), análisis de lenguaje (lenguaje), fotografía (estética), valores transmitidos (ideología y valores), feedback / interacción (difusión).

\section{Discusión y conclusiones}

Los instagramers que fueron objeto de análisis a través de sus publicaciones en Instagram buscaban generar en todos los casos cercanía con sus seguidores, al mostrar fotos de su vida diaria, viajes, familia, mascotas, pasiones, aficiones, entretenimientos, consejos e inclusive incluyen algo de humor, buscan que los seguidores observen que son personas como ellos que pueden caer y levantarse que pese a gozar de fama también tienen un hogar, una afición y son alguien más que lo que se ve en televisión o en medios de comunicación en general.

El lenguaje utilizado por los instagramers analizados en la mayoría de los casos es natural, claro y con frases cortas, se observa el uso de hashtags \# con palabras que hacen referencia a viajes, tiendas de ropa o simplemente sus lemas de vida. En otros casos hacen uso del humor para acompañar sus fotografías y captar así la atención de sus seguidores.

Si de llamar a la participación de los seguidores se trata los instagramers analizados saben cómo hacerlo, pues ellos captan la atención de su audiencia a colocar me gusta y comentar sus fotografías a cambio de darles como respuesta un "hola" a sus comentarios. Además plantean preguntas como: "¿qué les parece mi nuevo look? ¿desean que salga ahora mi nuevo álbum? ¿cómo me veo hoy? Comenta...", "Le daré like al último me gusta de esta publicación," entre otros tantos. Y los seguidores buscan que su nombre aparezca entre la lista de comentarios de su instagramer de preferencia. Este agrado de recibir una retroalimentación puede deberse a la influencia de los likes que tiene Instagram en el cerebro como fue demostrado por Sherman, Greenfield, Hernandez, y Dapretto (2017) en el estudio en el cual los participantes sentían una atracción especial por aquellas fotografías que habían recibido mayor número de "me gusta", las cuales provocaron mayor actividad en múltiples regiones cerebrales.

Uno de los principales problemas encontrados fue el determinar los instagramers más exitosos a nivel de Ecuador porque se observó la existencia de algunas herramientas utilizadas para la adquisición de datos automatizados en redes sociales; sin embargo, las mismas eran de pago por lo que se reali- 
zó una intensa búsqueda en Internet y se obtuvo datos de algunos rankings prestigiosos sobre redes sociales y blogs como es Alianzo Ranking, el cual ha sido la herramienta principal de recolección de datos. Se considera que de no haber existido la limitación económica hubiese sido de gran interés el poder emplear alguna otra herramienta de medición de éxito de instagramers para así lograr determinar la posible existencia de desviaciones entre ellas.

\section{Bibliografía}

Berrocal, S., Campos-Domínguez, E., \& Redondo, M. (2014). Prosumidores mediáticos en la comunicación política: El 'politainment' en YouTube. Comunicar, 43, 65-72.

Colliander, J., \& Marder, B. (2018). "snap happy” brands: Increasing publicity effectiveness through a snapshot aesthetic when marketing a brand on Instagram. Computers in Human Behavior, 78, 34-43. https://doi. org/10.1016/j.chb.2017.09.015

Castells, M. (2009). Communication Power. Oxford: Oxford University Press.

Djafarova, E., \& Rushworth, C. (2017). Exploring the credibility of online celebrities' Instagram profiles in influencing the purchase decisions of young female users. Computers in Human Behavior, 68, 1-7. https://doi. org/10.1016/j.chb.2016.11.009

Jin, S. V., \& Muqaddam, A. (2018). Narcissism 2.0! Would narcissists follow fellow narcissists on Instagram?" the mediating effects of narcissists personality similarity and envy, and the moderating effects of popularity. Computers in Human Behavior, 81, 31-41. https://doi.org/10.1016/j.chb.2017.11.042

Lacoste, J. A. (1998). Internet: la tecnología al servicio de la prevención. Revista Comunicar, 10,181-186.

Moya-Sánchez, M., \& Herrera-Damas, S. (2016). Cómo medir el potencial persuasivo en twitter: Propuesta metodológica. Palabra Clave, 19(3), 838-867. https://doi.org/10.5294/pacla.2016.19.3.7

Pittman, M., \& Reich, B. (2016). Social media and loneliness: Why an Instagram picture may be worth more than a thousand Twitter words. Computers in Human Behavior, 62, 155-167.https://doi.org/10.1016/j.chb.2016.03.084

Ritzer, G., Dean, P., \& Jurgenson, N. (2012). The Coming of Age of the Prosumer. American Behavioral Scientist, 56(4), 379-398. https://doi. org/10.1177/0002764211429368 
Romero-Rodríguez, L., \& Aguaded, I. (2016). Consumo informativo y competencias digitales de estudiantes de periodismo de Colombia, Perú y Venezuela. Convergencia. Revista de Ciencias Sociales, 23(70), 35-57.

Santoveña, S. M. (2011). Incidencia de los nuevos alfabetismos en la mejora de la calidad de la enseñanza : el caso de los blogs. Aula Abierta, 39(2), 59-68.

Schwienbacher, I., Fendt, M., Richardson, R., \& Schnitzler, H. (2004). Temporary inactivation of the nucleus accumbens disrupts acquisition and expression of fear-potentiated startle in rats. Brain Research, 1027(1-2), 87-93. https://doi.org/10.1016/j.brainres.2004.08.037

Sheldon, P., \& Bryant, K. (2016). Instagram: Motives for its use and relationship to narcissism and contextual age. Computers in Human Behavior, 58, 8997. https://doi.org/10.1016/j.chb.2015.12.059

Sherman, L. E., Greenfield, P. M., Hernandez, L. M., \& Dapretto, M. (2017). Peer Influence Via Instagram: Effects on Brain and Behavior in Adolescence and Young Adulthood. Child Development, 89(1), 37-47. https://doi. org/10.1111/cdev.12838

Tejedor, S., Campo, I., Esquivel, F., Gaviria, J. G., Giraldo, S., Larrondo, A.,... Rodrigues, P. (2009). Ciberperiodismo-Libro de estilo para ciberperiodistas. Santo Domingo, República Dominicana: Editorial ITLA.

Toffler, A. (1970). Future shock. New York: Bantman Book.

Utz, S., Tanis, M., \& Vermeulen, I. (2012). It Is All About Being Popular: The Effects of Need for Popularity on Social Network Site Use. Cyberpsychology, Behavior, and Social Networking, 15(1), 37-42. https://doi. org/10.1089/cyber.2010.0651

Yau, J. C., \& Reich, S. M. (2018). "It's Just a Lot of Work": Adolescents' SelfPresentation Norms and Practices on Facebook and Instagram. Journal of Research on Adolescence, 29(1), 196-209. Recuperado de: https://doi. org/10.1111/jora.12376

Wang, D., Xiang, Z., \& Fesenmaier, D. R. (2016). Smartphone Use in Everyday Life and Travel. Journal of Travel Research, 55(1), 52-63. https://doi. org/10.1177/0047287514535847

Fecha de envío: 2019/0I/31; Fecha de aceptación: 2019/07/25;

Fecha de publicación: 2019/09/01 